\title{
Three Dimensional Simulation of Grain Growth in the Presence of Mobile Pores
}

\author{
Veena Tikare \\ Sandia National Laboratories \\ Albuquerque, NM 87185-1411 \\ Mark A. Miodownik \\ ${ }^{2}$ Mechanical Engineering Department, University College Dublin \\ Belfield, Dublin, Ireland. \\ and \\ Elizabeth A. Holm \\ Sandia National Laboratories \\ Albuquerque, NM 87185-1411
}

\begin{abstract}
A kinetic, three dimensional, Monte Carlo model for simulating grain growth in the presence of mobile pores is presented. The model was used to study grain growth and pore migration by surface diffusion in an idealized geometry that ensures constant driving force for grain growth. The driving forces, pore size and pore mobilities were varied to study their effects on grain boundary mobility and grain growth. The simulations captured a variety of complex behaviors including reduced grain boundary velocity due to pore drag that has been predicted by analytical theories. The model is capable of treating far more complex geometries including polycrystals. We present the capabilities of this model and also discuss its limitations.
\end{abstract}

\section{Introduction}

During the later stages of sintering of ceramic powders, pores become isolated and pressurized. Densification is accompanied by curvature driven grain boundary migration, which causes grain growth. Under certain circumstances, the pores become mobile and attach themselves to the moving grain boundaries. These pores at the grain boundaries exert a pinning force on the grain boundaries, modifiying their velocity. In extreme cases, the pores can completely stagnate grain growth (Zener pinning). The extent of this pinning behavior is influenced by the pore size, shape and mobility as well as by the grain size and grain boundary mobility ${ }^{1,2,3,4}$. Predicting the microstructural evolution of such systems is very complex and has enjoyed limited ${ }^{4}$.

Grain growth in the presence of a mobile second phase has been studied in twodimensions by Tikare and Holm ${ }^{5}$. They found grain growth to be Zener pinned. When two pores come into contact with each other by random walk, they immediately coalesced to form a single pore. This allowed the local grains that were previously pinned by the pores to grow until the new distribution of pores around them pinned them again. Thus grain growth was rate limited by the pore migration and so both pore growth and grain growth had a growth exponent of $\mathrm{n}=5$, which is consistent with the random walk and coalescence mechanism. 


\section{DISCLAIMER}

This report was prepared as an account of work sponsored by an agency of the United States Government. Neither the United States Government nor any agency thereof, nor any of their employees, make any warranty, express or implied, or assumes any legal liability or responsibility for the accuracy, completeness, or usefulness of any information, apparatus, product, or process disclosed, or represents that its use would not infringe privately owned rights. Reference herein to any specific commercial product, process, or service by trade name, trademark, manufacturer, or otherwise does not necessarily constitute or imply its endorsement, recommendation, or favoring by the United States Government or any agency thereof. The views and opinions of authors expressed herein do not necessarily state or reflect those of the United States Government or any agency thereof. 


\section{DISCLAIMER}

Portions of this document may be illegible in electronic image products. Images are produced from the best available original document. 
It is known, however, that 2-D and 3-D Zener pinning are completely different phenomena. A 3-D boundary interacting with an array of pores can be treated as a single thermodynamic entity. The velocity of such a boundary can therefore be calculated by summing the pinning forces and driving forces acting on it. Boundary detachment from pores depends only on the net force acting on the boundary. However when a 2-D boundary meets a pore, it effectively splits into two independent boundary segments, each of which can satisfy the equilibrium surface tension required at the pore interface. The point at which each segment attaches to the pore effectively becomes a node. Detachment from the pore can only occur if the two boundary segments rotate by an angle of 180 degrees. This was first pointed out by Hillert ${ }^{6}$, who showed that this results in the stabilization of grains with less than 6 neighbors, leading to a radically different pinning behavior. This is the crucial difference between 2-D and 3-D pinning. In 3-D, the pinning force is a frictional force due to the local increase in boundary area during pore detachment. In $2-\mathrm{D}$, pinning arises from the topological consideration that the pores act as static nodes. The upshot of this is that one cannot use 2-D models to investigate the pinning behavior of 3-D polycrystals.

In a recent paper Miodownik et al. ${ }^{7}$ showed that a 3-D Monte Carlo Potts model can be used to accurately simulate Zener pinning. In this paper, we use a similar model to investigate the effect of mobile pores on a single mobile grain boundary. The advantage of the model over analytical approaches is that it can incorporate geometric complexity of real microstructures and the accompanying thermodynamic and kinetic factors. While the model is presented here as grain growth in the presence of isolated, mobile pores, it is readily applicable to grain growth in the presence of a mobile second phase that is either isolated or interconnected.

\section{Development of the Simulation Method Description of the Model}

The Potts $\mathrm{Model}^{8}$, a kinetic Monte Carlo model, was used to simulate grain growth and pore migration. In the Potts model, a canonical ensemble of grain sites and pore sites is allowed to populate a cubic lattice. These grain and pore sites may be conceptualized as discrete, arbitrary quanta of matter, on the scale of billions of atoms, belonging to a grain or to a pore. Thus all information about atomic interactions is lost. The grain sites can assume one of $Q$ distinct, degenerate states, where $q=[1,2, \ldots Q]$. The pore sites can assume only one state, $q=-1$. Contiguous grain sites of the same state $q$ form a grain and contiguous pore sites form a pore. Grain boundaries exist between neighboring grain sites of different states, $\boldsymbol{q}$, and pore-grain interfaces exist between neighboring pore and grain sites. Grain boundaries and pore-grain interfaces have an excess energy associated with them. The total system energy is the sum of this excess interfacial energy, given by the Hamiltonian:

$$
E=\frac{1}{2} \sum_{i=1}^{N} \sum_{j=1}^{26}\left(1-\delta\left(q_{i}, q_{j}\right)\right)
$$


where $N$ is the total number of sites, $\delta$ is the Kronecker delta with $\delta\left(q_{i}=q_{j}\right)=1$ and $\delta\left(q_{i}\right.$ $\left.\neq q_{j}\right)=0, q_{i}$ is the state of the grain or pore at site $i$ and $q_{j}$ is the state of one of the 26 first, second and third nearest neighbors at site $j$. Thus, the only energy considered in the simulation is the interfacial energy and all unlike neighbors contribute one arbitrary unit of energy to the system. As pore sites can assume only one state, $q=-1$, there are no pore boundaries and all pores sites will coalesce. In contrast, grain sites can assume many different states making grain boundaries possible. This yields a two-component, two-phase system with uniform, isotropic interfacial energies between grains and between grains and pores.

Grain growth is simulated using the method developed in previous works ${ }^{9,10,11}$. First a grain site is chosen at random from the simulation space. Then a new state $q$ is chosen at random from the $Q$ possible states in the system. The change in energy, $\Delta E$, is calculated using eq. 1, and the probability $\boldsymbol{P}(\Delta \boldsymbol{E})$ that the site will change orientation is then determined from the transition probability function:

$$
P=\left\{\begin{array}{ccc}
\exp \left(\frac{-\Delta E}{k_{B} T}\right) & \text { for } & \Delta E>0 \\
1 & \text { for } & \Delta E \leq 0
\end{array}\right.
$$

\section{Equation 2}

Where $k_{B} T$ is the simulation temperature and defines the thermal fluctuation of the simulation. The standard Metropolis algorithm is used to accept changes. A random number, $\boldsymbol{R}$, between $\mathbf{0}$ and $\mathbf{1}$ is generated. If the $\boldsymbol{R} \leq \boldsymbol{P}$, then the grain growth step is accepted, if not, the original state is restored. The simulation temperature used for grain growth varied from $k_{B} T=0$ to 2.0 .

Pore migration is simulated using conserved dynamics, so that the total number of pore sites and grain sites remains constant throughout the simulation. First a pore site is chosen at random and next a neighboring grain site is chosen at random. The two sites are temporarily exchanged with the grain site assuming a new state $q$. The new state $q$ is chosen, so that it results in the lowest energy. The change in energy for this exchange is calculated using eq. 1 and again the standard Metropolis algorithm is used to perform the pore migration step using eq. 2 to determine the transition probability. This algorithm has been shown to simulate pore migration by surface diffusion ${ }^{5}$.

Time in the Potts model is measured in units of Monte Carlo step; 1MCS corresponds to $N$ attempted changes where $N$ is the total number of sites in the system. The mobility ratio of pore boundaries to grain boundaries is varied by varying the attempted grain growth step to pore migration steps. Thus, at pore site mobility $M=1$, the grain sites and pores sites have attempted equal number of changes and at $M=10$, the pore sites have attempted 10 pore migration steps for each grain growth attempt. 


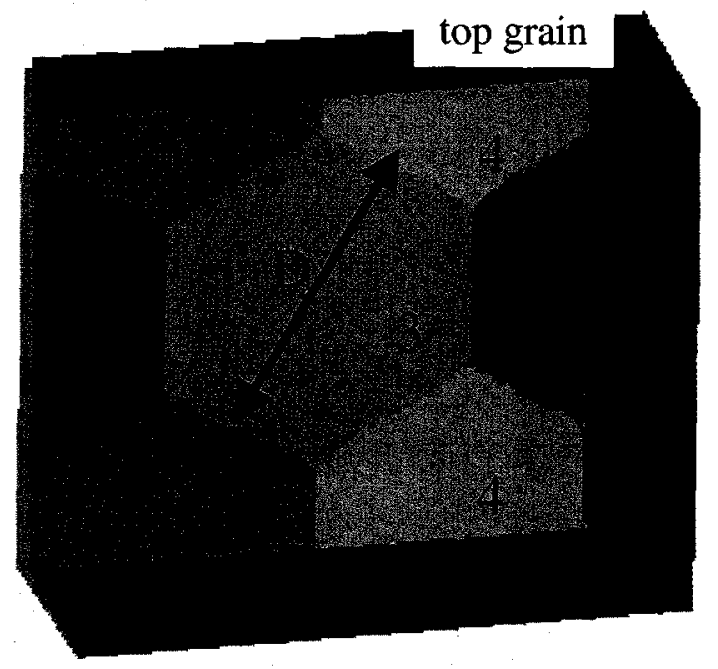

Figure 1. The geometry used to study grain growth with constant driving force consisted of four columnar grains, numbered 1 to 4 , with hexagonal cross-section with a flat grain placed over them (the top grain has been partially cut-away to show the four columnar grains).

\section{3-D Hexagonal Geometry}

To understand and characterize the motion of both grain boundaries and pores, we studied a simple geometry constructed to ensure that a particular grain boundary would move with constant velocity in a given direction. This geometry was developed by Miodownik et $\mathrm{al}^{7}$ to study Zener pinning. This geometry, shown in figure 1 , consists of four columnar grains with hexagonal cross-sections with one flat grain placed above them. Periodic boundary conditions are used in the $\boldsymbol{X}$ - and $\boldsymbol{Y}$-directions so that the four columnar grains are identical in shape and size. Only the boundary between the planar grain and the hexagonal grains is in a non-equilibrium state, which results in a driving force for motion in the Z-direction. The driving force, $\boldsymbol{F}_{\boldsymbol{d}}$, is given by the rate of change of grain boundary energy, $\boldsymbol{A} \gamma$, with respect to grain volume, $\boldsymbol{V}$, as the grain grows in the $Z$-direction. Assuming $\gamma$ is constant, the driving force is:

$$
F_{d}=\frac{d A / d Z}{d V / d Z} \gamma=\frac{16 D / \sqrt{3}}{8 D^{2} / \sqrt{3}} \gamma=\frac{2 \gamma}{D}
$$

Equation 3

where $\boldsymbol{D}$ is the grain size shown in Figure 1 and $\gamma$ is the surface energy. Since the grain size $\boldsymbol{D}$ remains constant, the driving force $\boldsymbol{F}_{\boldsymbol{d}}$ also remains constant during grain growth in this geometry. Assuming the velocity of the grain boundary $v$ is proportional to the driving force, we can write:

$$
v=\mu F_{d}=\frac{2 \gamma \mu}{D}
$$

\section{Equation 4}


where $\mu$ is the grain boundary mobility. Thus, the geometry shown in figure 1 allows us to vary the driving force for grain growth and grain boundary velocity simply by changing the grain size $\boldsymbol{D}$. Miodownik ${ }^{7}$ et al. have verified that the model behaves according to equation 4 .

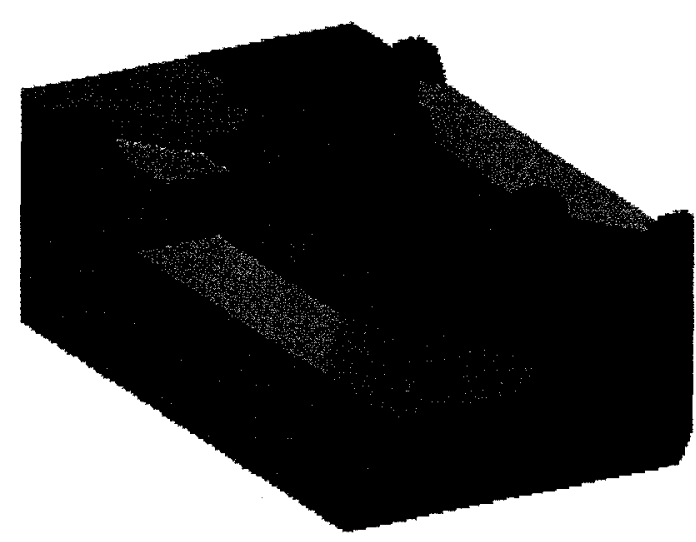

Figure 2a. Spherical pores are placed at the quadra-junctions. The top grain is not shown and cutout shows that the crosssections are circular.

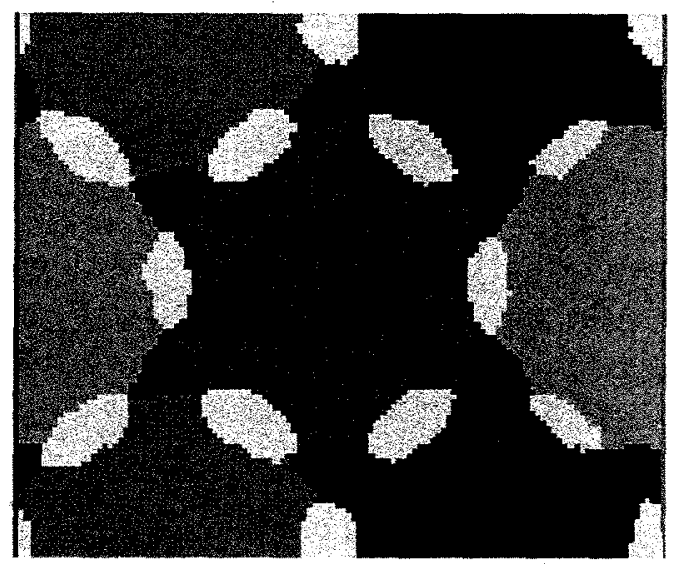

Figure 2c. Slice through the microstructure showing the triangular cross-section of the pores.

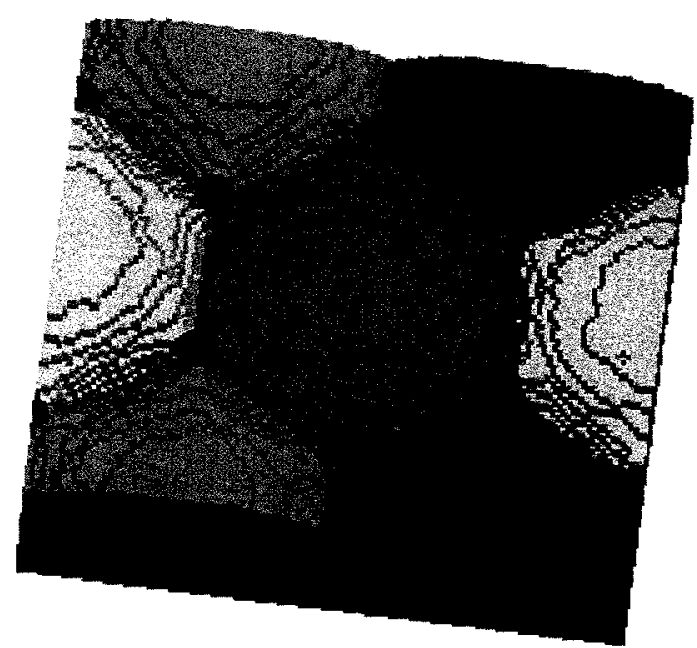

Figure 2b. The minimum energy shape of the grain boundary.

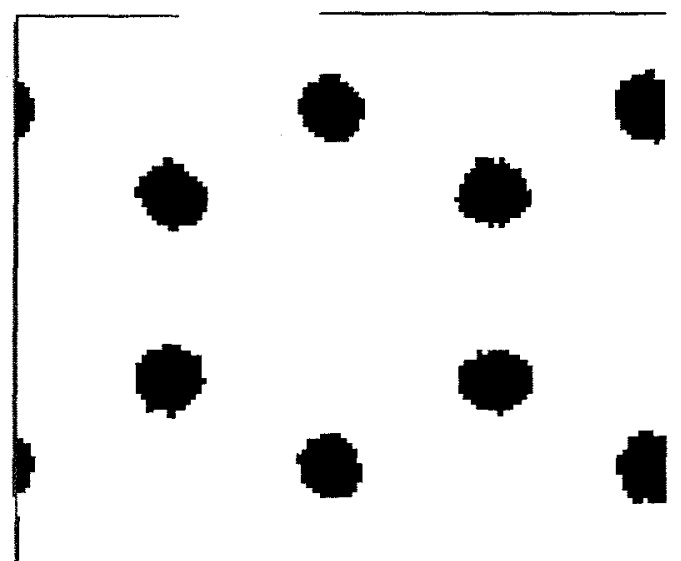

Figure 2d. Slice through the top grain showing the circular cross-section of pores.

Grain growth in the presence of mobile pores was simulated by placing spherical pores at all the quadra-junctions as shown in figure $2 \mathrm{a}$. Pores were placed at the quadra-junctions rather than at grain centers because pores placed at the grain centers migrated to the grain junctions as these are their minimum energy locations. Furthermore, more than one pore would often migrate to the same quadra-junction, leaving other junctions without pores. 
Thus, to maintain a constant driving force, pores of the same size were placed at the quadra-junctions. Once the simulation is started, the pores and grain boundary shapes change to their minimum energy shapes. The shape of the grain boundary is a dome of constant curvature as shown in figure $2 \mathrm{~b}$. The equilibrium shape of the pores is very similar to an ice-cream cone with the cone having a triangular cross-section between its three adjacent, columnar grains. Figure $2 \mathrm{c}$ shows the triangular cross-section of the pore between three grains and figure $2 d$ shows the circular cross-section (like the ice cream on top of the cone) protruding into the top grain. The triangular and circular cross-sections, shown in figures $2 c$ and $d$, are not smooth and regular as is characteristic of Monte Carlo simulation at temperatures $k_{B} T>0$ due to thermal roughening of the surfaces. Before the grain boundary and pores reach their minimum energy shape, the velocity of the grain boundary fluctuates as shown in figure 3 , a plot of grain boundary velocity as a function of position. Once their minimum energy shape is formed, the velocity becomes constant. The grain boundary and pores retain their minimum energy shape in the constant velocity portion of the simulation. When the grain boundary and pores intersect the edge of the simulation, the velocity becomes erratic. It is the constant velocity portion of the simulation that is useful, as the driving force remains constant during this portion of the simulation. Finite simulation temperatures must be used for both grain growth and pore

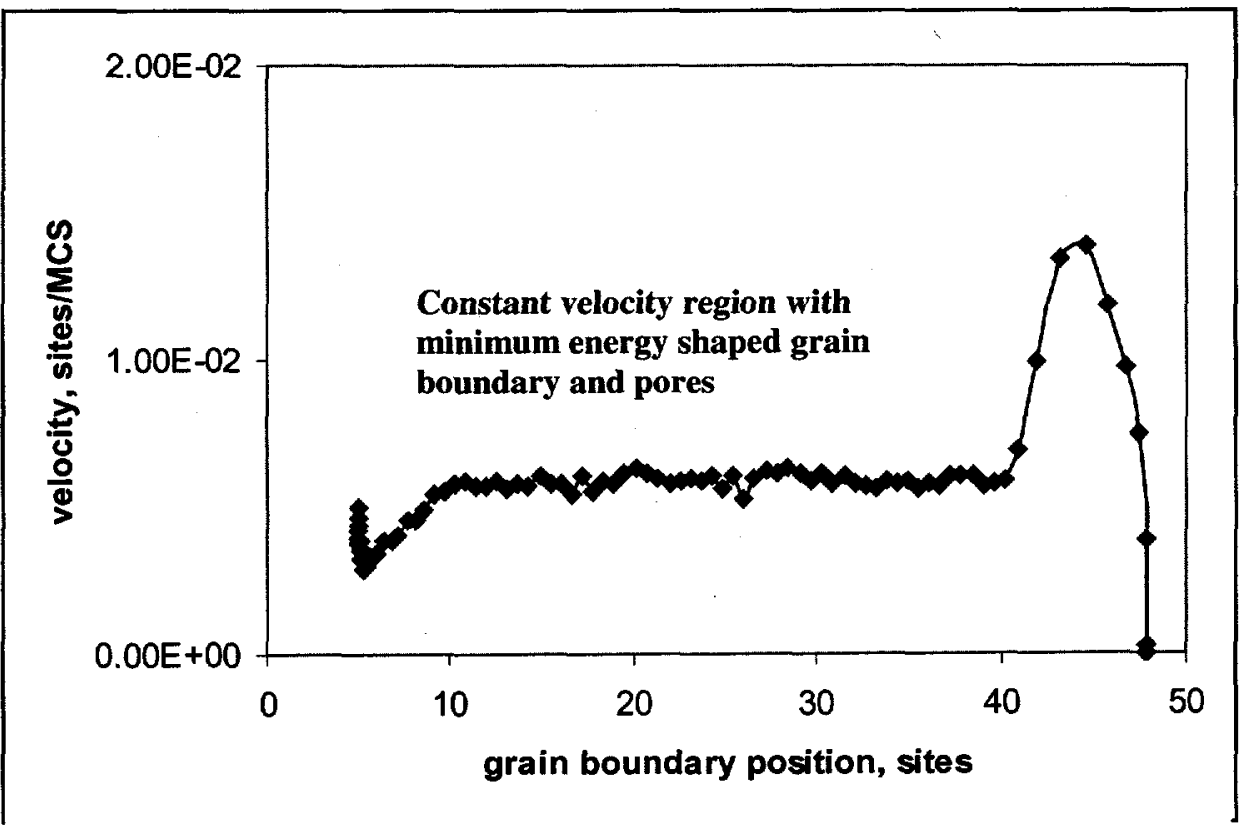

Figure 3. Plot of grain boundary velocity as a function of position for the case of grain size $D=58$, pore size $d=6$, pore to grain boundary mobility ratio $M=5$, grain growth temperature $k_{B} T_{g}=1.0$ and pore migration temperature $k_{B} T_{p}=2.0$

migration to correctly simulate grain growth in the presence of a mobile phase. A complete discussion of the need for finite simulation temperature follows in the Appendix. In this work, we used pore migration temperature $k_{B} T_{p}=1.0$ and grain growth temperature $k_{B} T_{g}=1.0$ to study grain growth in the presence of mobile pores. 


\section{Results}

Grain growth in the presence of pores of different sizes and mobilities was investigated. The pore size was varied from $d=4.5$ to 6 (corresponding to pore volume of 50 to 125 sites) and the pore to grain boundary mobility ratio was varied from $M=1$ to 20 . The grain boundary velocity for different pore mobilities and constant pore size, $d=6$, is plotted as a function of driving force in figure 4a. The solid line is grain boundary velocity for normal grain growth with no pores. The data represented by symbols is the

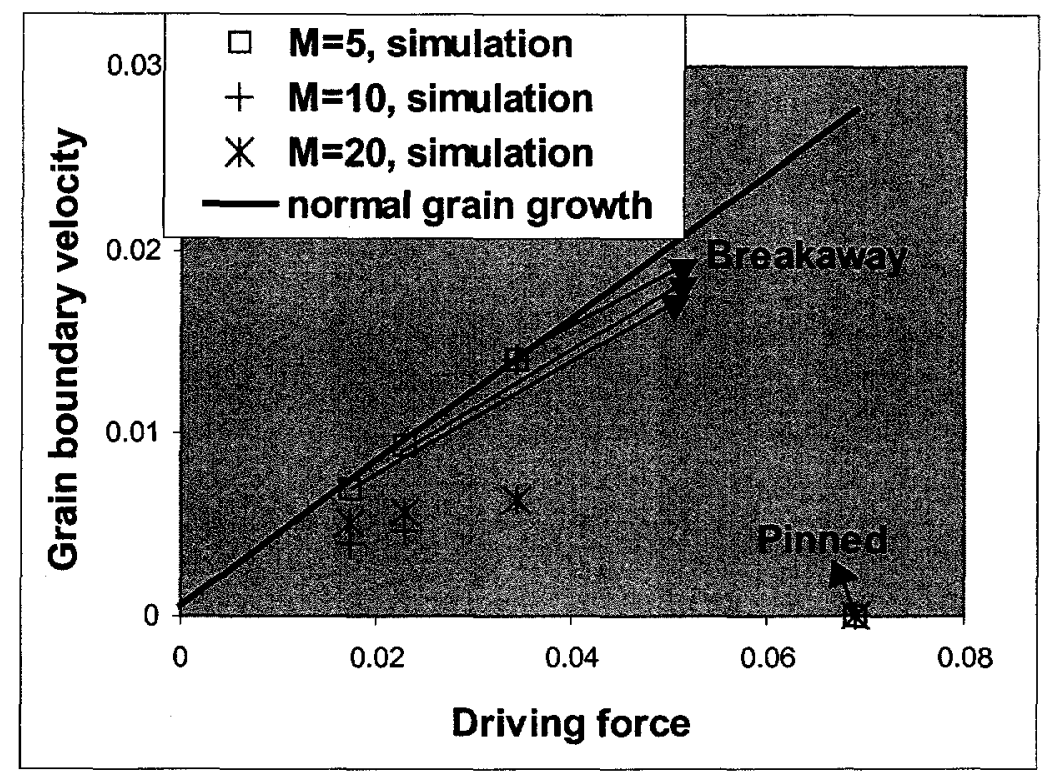

Figure 4a. Grain boundary velocity as a function of driving force for pores of constant size $d=6$ and varying mobilities.

grain boundary velocity with mobile pores at all the quadra-junctions. At the low pore site mobility, $\boldsymbol{M}=1$ to 5 , the grain boundary broke away from the pores for all driving forces considered. When $\mathrm{M}=10$, the pores moved with the grain boundary at low driving forces but broke away at high driving forces. The same general behavior was anticipated for $M=20$ but break-away was never observed. The reason for this is that high driving force requires small values for $\mathrm{D}$. When $\mathrm{D}$ starts to approach the size of the cells in the simulations then anomalous results are observed due to lattice effects: the curvature cannot be properly resolved at these sizes and is distorted to the point where the grains have flat surfaces as shown in figure $4 \mathrm{~b}$. This leads to full pinning of the grains by the pores and is an artifact of the simulation technique.

Grain growth was studied as a function of driving force for constant pore site mobility $M$ $=10$ and two different pore sizes, $d=4.5$ and 6 . The results are shown in figure 5. At the small pore size $d=4.5$, the grain boundary velocity was reduced by the presence of the pore, but break-away did not occur. At the larger pore size of $d=6$, the grain boundary velocity was reduced further and break-away occurred at high driving force where the pores impeded grain boundary mobility too much. 
In summary, the presence of mobile pores modified grain growth behavior tremendously and a variety of phenomena was observed. The shape of the grain boundary is modified by the presence of pores. In general, as the effective pore mobility is decreased or the pore size increased, the grain boundary velocity decreases. However, break-away can occur if the boundary is impeded too much. Complete pinning of the grain boundary by the pores was observed but this is a lattice effect.

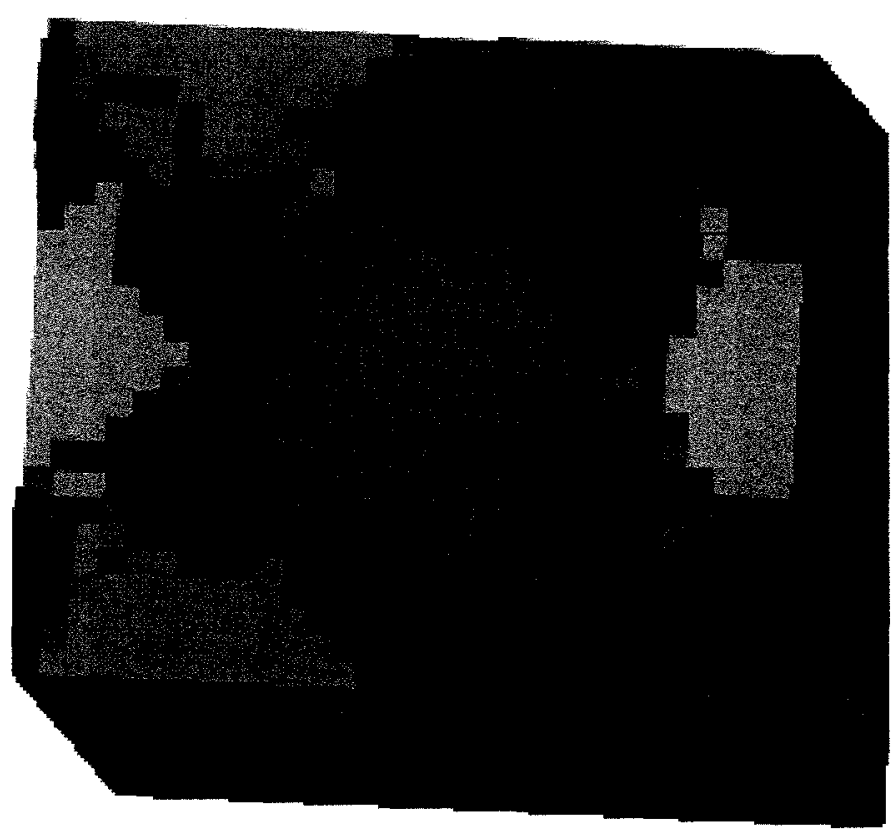

Figure 4b. The microstructure for grain size $D=14$ and pore size $d$ $=6$. The large pores in small grains distort the grain boundary curvature.

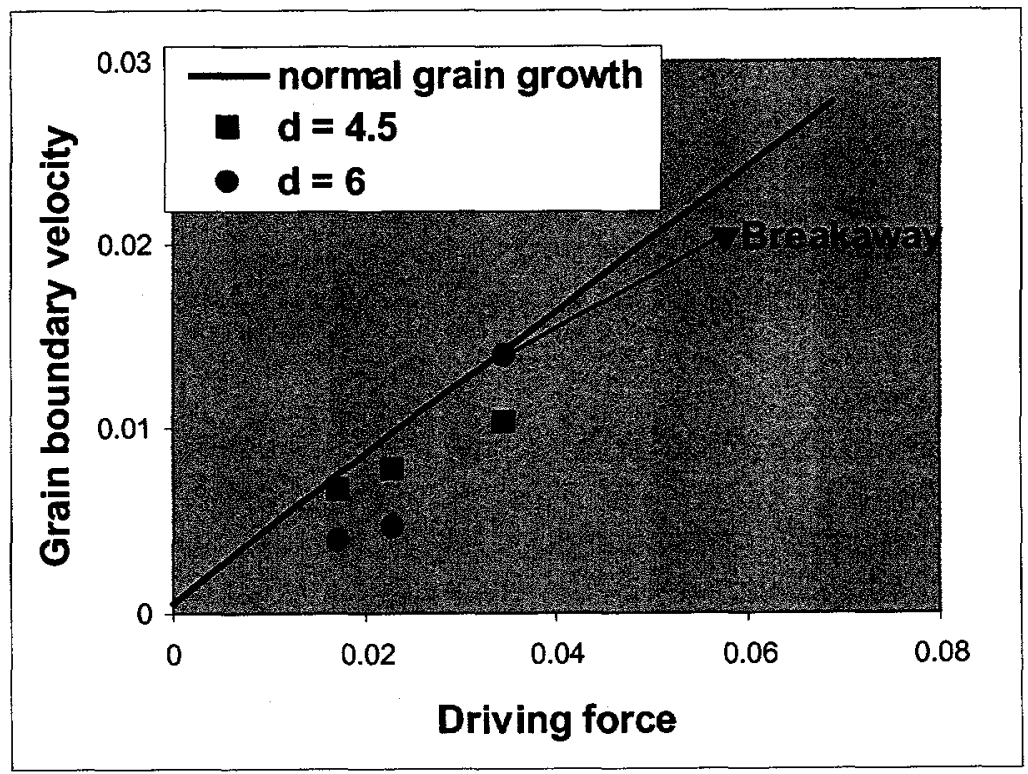

Figure 5. Grain boundary velocity as a function of driving force for pores of constant mobility and different pore sizes. 


\section{Discussion}

Gottstein and Shvindlerman ${ }^{12}$ developed an analytical model of grain boundary motion in the presence of mobile particles. The theory is very similar to the classic work of Cahn ${ }^{13}$ and Lucke and Stuwe ${ }^{14}$ for grain boundary motion with impurity drag. They proposed the velocity of a pore-laden grain boundary is

$$
\boldsymbol{v}=\mu\left(\boldsymbol{F}_{d}-\boldsymbol{F}_{i}\right)
$$

Equation 5

where $F_{i}$ is the drag force due to particles and is a function of interaction energy between the particles and the grain boundary, the concentration of particles, their mobility and the grain boundary velocity. There are two limiting cases; high particle mobility when the boundary velocity is determined by the mobility of the boundary, and low particle mobility when the velocity is determined by the mobility of the particles. The velocities in between these two extremes are difficult to predict because velocity is specific to the details of each case. However, the driving force for transition from one branch to the other was estimated to occur at the Zener pinning condition of the particles.

We consider pores to behave identically to particles and thus the velocity of the pore and therefore the boundary is given by

$$
v_{p}=\mu_{p} F_{p}
$$

Equation 6

where $\mu_{p}$ is the effective mobility of the pore. At steady state the velocity of the grain boundary and pore are the same, therefore by substituting equation 6 into equation 5 and setting $v=v_{p}$, we get

$$
v=\frac{\mu F_{d}}{1+\frac{\mu}{\mu_{p}}}
$$

\section{Equation 7}

The effective pore mobility depends strongly on pore size. In the case of spherical pores, it has been shown ${ }^{2}$ to be proportional to $D_{i} d^{4}$, where $D_{i}$ is the interfacial diffusion coefficient and $\boldsymbol{d}$ is the pore size. In this simulation, pore site mobility $\boldsymbol{M}$ is proportional to $D_{i}$ and so we get

$$
v=\frac{\mu F_{d}}{1+\frac{\mu l d^{4}}{M}}
$$

\section{Equation 8}

where $l$ is a proportionality constant.

The velocities of pore-laden grain boundaries, given by equation 8 , are plotted in figures $6 \mathrm{a}$ and $\mathrm{b}$. The proportionality constant $l$ used in all plots is $l=7$ and was determined by best fit. The grain boundary velocity predicted by equation 8 agrees with the trend that grain boundary velocity increases with increasing driving force, increasing pore mobility and decreasing pore size. However, exact agreement was not obtained. We attribute the difference to two factors. First, the velocity predicted by equation 8 assumes pores are spherical. However, pores are not spherical as shown in figures $2 \mathrm{c}$ and $\mathrm{d}$. Second, the curvature of the grain boundary is assumed to be the same as the curvature of the grain boundary with no pores. However, the presence of pores at the quadra-junctions does 
change the curvature of the grain boundary. The grain boundary curvature decreases as the relative size of the pore with respect to the grain size increases. This decrease in grain boundary curvature is expected to result in lower grain boundary velocity as observed in the simulations. Furthermore Rödel and Glaeser ${ }^{4}$ found evidence for these effects in polycrystalline alumina. They joined single crystal alumina to polycrystalline

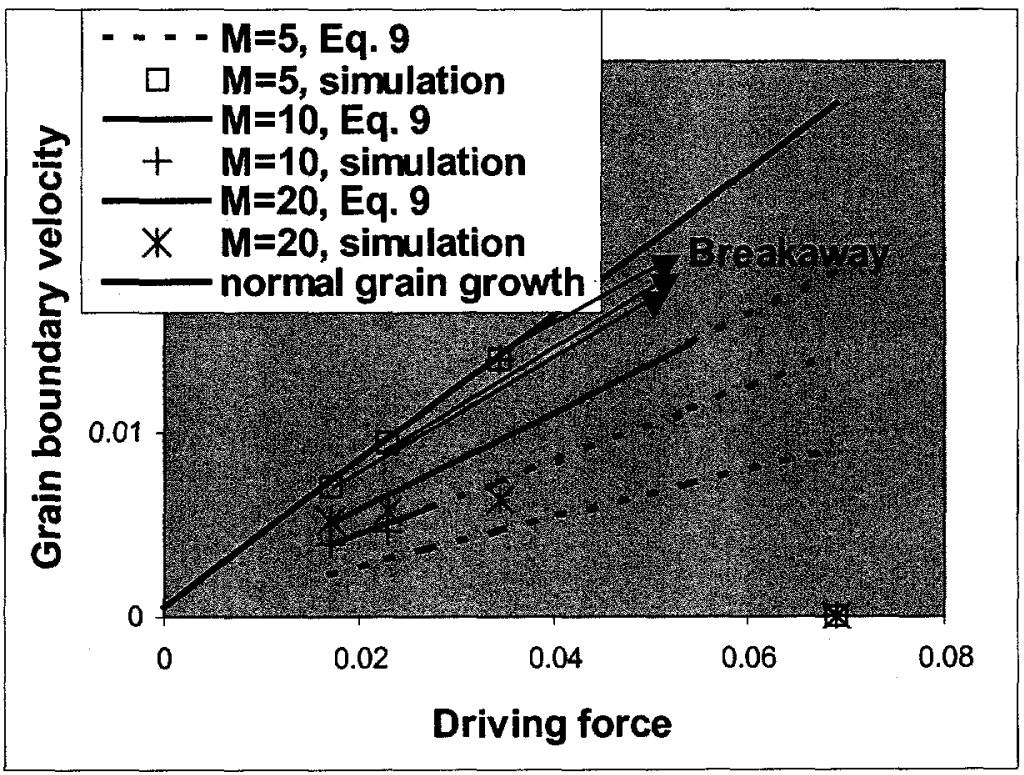

Figure 6a. The simulation results are compared to analysis. The solid lines are pore laden grain boundary velocities for pores of size $d=6$ with different mobilities. The dotted line represents driving forces at which grain boundary break-away is predicted by equation 10 .

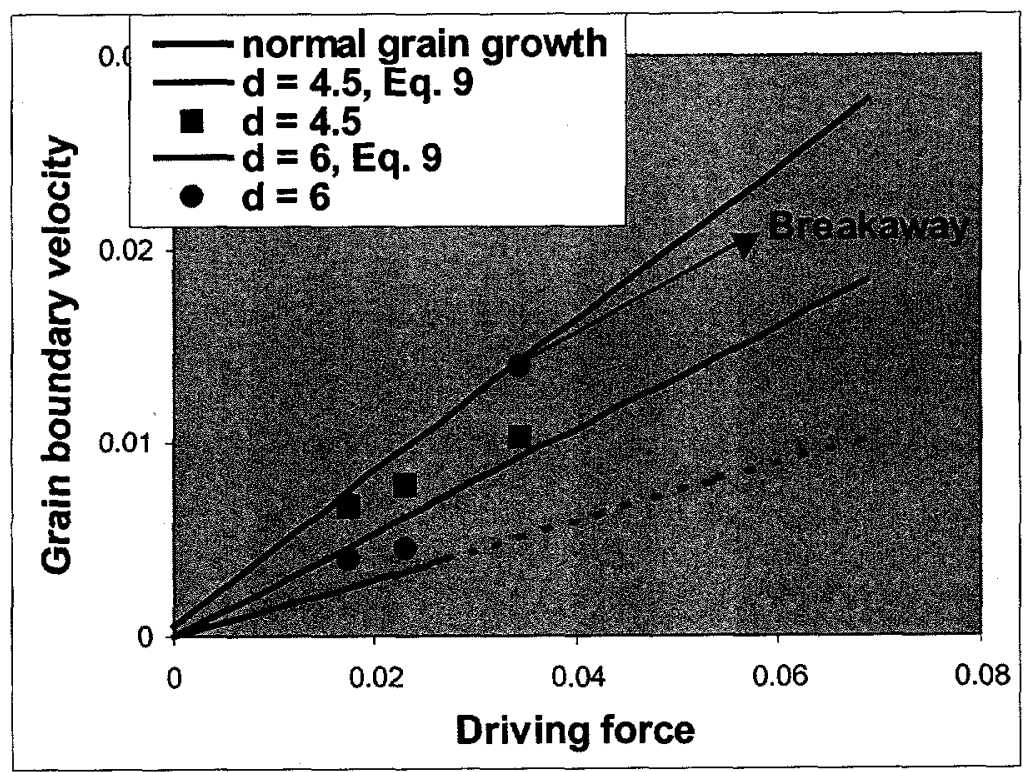

Figure 6b. The simulation results are compared analysis. The solid lines are pore laden grain boundary velocities for pores of size $d=6$ with different mobilities. The dotted line represents driving forces at which grain boundary break-away is predicted by equation 10 . 
alumina and induced array of pores at the interface. The first stage of microstructural evolution was characterized by the pores at the interface changing their shape to a minimum energy configuration. Pores at quadra-junctions assumed the ice-cream cone shape similar to those observed in our simulations. They reported pore-laden grain boundary velocity increased with increasing surface diffusivity. This is the same as the results observed in this work, where the velocity increased with increasing pore site mobility $\boldsymbol{M}$. They also reported that the grain boundary separated from the pores when the polycrystalline grain size was small as observed in our simulations. This behavior is predicted by Gottstein and Shvindlerman ${ }^{12}$. The maximum drag force that a pore can apply can be estimated to be its Zener force $F_{Z}$, the force exerted by a stationary pore on the grain boundary

$$
F_{z}=g d
$$

Equation 9

and $\boldsymbol{g}$ is a proportionality constant for the geometry considered in this study. From equations 6,7 and 9, the grain boundary will break away from a pore when

$$
F_{d}=g d+\frac{g l M}{\mu d^{3}}
$$

Equation 10

Equation 10 predicts that the break-away force increases with increasing pore site mobility as observed in our simulations. The relationship between pore diameter and break-away force is more complicated and depends critically on the value of $I M / \mu$. If $\boldsymbol{l} M / \boldsymbol{\mu}>\boldsymbol{d}$, then larger particles lead to smaller break-away forces as observed in these simulations.

The driving force for break-away given by equation 10 is also shown in figures $6 a \mathrm{a}$ b. The proportionality constant $\boldsymbol{g}$ used to calculate the break-away driving force is $\boldsymbol{g}=$ 0.0003 and was determined by best fit to the data. The break-away driving force predicted by equation 10 increases with increasing pore mobility and decreasing pore size and is in good agreement with simulation results.

Previous work ${ }^{13,14,12}$ in theory of grain boundary drag predicts a hysteresis in the velocity - driving force relationships between the two branches of grain boundary velocity. It was not possible in this study to characterize the hysteresis as it was not possible to change the driving force (i.e. change grain size $D$ ) continuously in a single simulation. However, unlike the analytical theories that assume no change in the shape and distribution of the drag phase, the model used here is able to simulate the microstructural changes that occur when a grain boundary encounters the pores or detaches from a pore. This ability of the model to simulate the local changes in grain boundary and pore shape is shown in figures $2 \mathrm{a}-\mathrm{d}$ and the effect it has on the grain boundary velocity is shown in the initial stage of figure 3 .

While the model is capable of incorporating all the physics necessary to properly simulate grain growth in the presence of pores, several artifacts were observed. Finite simulation temperatures for grain growth and pore migration must be used to ensure that artificial pinning, premature break-away and incorrect grain boundary velocities from non-equilibrium shaped microstructures do not result (see appendix). If the grain size is 
too small to resolve curvature of the grain boundaries, artificial pinning may be obtained as seen in figure $4 \mathrm{~b}$. Judicious application of the model will result in proper simulation of grain growth in the presence of mobile pores by incorporating all the necessary microstructural complexity.

The geometry used to simulate grain growth in the presence of mobile pores, was a highly controlled one, unlike the geometries encountered in typical polycrystalline materials. This geometry was designed to isolate variables contributing to the velocity of pore-laden grain boundaries and to grain boundary breaking away from pores. This controlled geometry was used to validate the model as well as to gain understanding of grain growth in the presence of pores. In a typical polycrystalline material, each grain boundary is expected to move at a different velocity related to its own curvature as well as the size and number of pores on that grain boundary. Some grain boundaries may break away from some pores and others may not. The model presented in this paper can be applied to typical polycrystalline geometries to study grain growth in the presence of mobile pores.

\section{Conclusions}

A kinetic, Monte Carlo model capable of simulating grain growth in the presence of mobile pores in three dimensions has been presented. The model simulated curvature driven grain growth by short range diffusion across grain boundaries and also pore migration by surface diffusion along the pore surfaces. The presence of mobile pores at the grain boundaries reduced grain boundary mobility by applying a drag force on the grain boundaries. The drag force applied by the pores to the grain boundary increased with increasing pore size and decreasing pore mobility. Thus, grain boundary velocity decreased with increasing pore size and decreasing pore mobility. However, grain boundaries did break away when the pore mobility becomes too small.

\section{Appendix}

Simulation Temperature

Potts model simulations of some microstructural evolution processes such as normal grain growth ${ }^{11}$ do not require finite temperature for proper simulation. However, simulation of most microstructural evolution process such as Zener pinning ${ }^{7}$, Ostwald ripening ${ }^{15}$, 2D grain growth in the presence of mobile pores ${ }^{5}$ and sintering ${ }^{16}$ require finite temperature for proper simulation. In this appendix, we show finite temperatures are necessary for proper simulation of 3D grain growth in the presence of mobile pores by evaluating the effect of temperature on the microstructural evolution. We do this by comparing the effect of simulation temperature on normal grain growth to grain growth in the presence of mobile pores. In both cases, the $3 \mathrm{D}$ hexagonal geometry is used.

The grain boundary velocities during normal grain growth with no pores are given in figure A1, a plot of grain boundary velocity as a function of driving force at different simulation temperatures $\boldsymbol{k}_{B} \boldsymbol{T}$. Figure A1 shows that grain boundary velocity is linearly proportional to the driving force as given by equation 4 and is good agreement with 


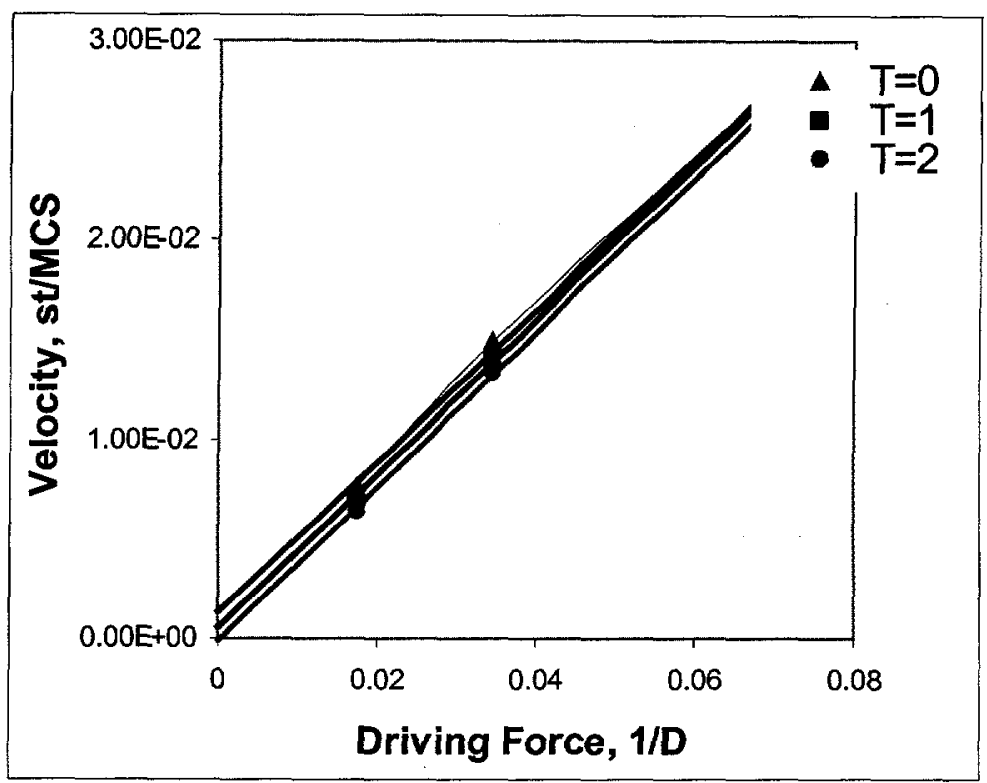

Figure A1. Grain boundary velocity for normal grain growth as a function of driving force at different grain growth temperatures. The symbols are data obtained from simulations and the straight lines are least-square fit through the data.

Miodownik et al's results ${ }^{7}$. Figure A1 also shows that the grain boundary velocity decreases slightly with increasing temperature as previously observed by Grest et al ${ }^{17}$ and Holm and Miodownik ${ }^{18}$. While there is a small dependence on simulation temperature, grain growth behavior is the same qualitatively and kinetically from $k_{B} T=0$ to 2 .

Grain growth in the presence of mobile pores were carried out for grain growth temperature ranging from $\boldsymbol{k}_{B} \boldsymbol{T}_{g}=0$ to 2 and pore migration temperatures ranging from $k_{B} T_{p}=0$ to 3. At grain growth temperature of $k_{B} T_{g}=0$ and pore migration temperature of $k_{B} T_{p}=0$, the grain boundary completely stagnated and was fully pinned by the pores at the quadra-junctions. Similar stagnation at simulation temperature $k_{B} T=0$, was observed both in the $2 \mathrm{D}$ simulation of grain growth with mobile pores ${ }^{5}$ (when $k_{B} T_{g}=0$ and $k_{B} T_{p}=0$ ) and in the 3D simulation of grain growth with stationary pinning particles ${ }^{7}$ (when $k_{B} T_{g}=0$ ). The stagnation, in all cases at $k_{B} T=0$, occurs because the grain growth can proceed only by continuously lowering its total energy given by equation 1 . However, for grain growth in the presence of mobile pores, the system must be able to locally increasing its energy in order to find the global lower energy states. If the simulation cannot sample these higher energy states, then stagnation occurs.

Finite temperature $k_{B} T_{g}>0$ was used for grain growth while holding the pore migration temperature at $k_{B} T_{p}=0$. In these simulations, the grain boundary was either completely pinned by large pores or the grain boundary broke away from small pores and normal grain growth occurred. The pores, however, did not move with the grain boundary at pore migration temperature $\boldsymbol{k}_{B} \boldsymbol{T}_{p}=0$. Next, finite temperature was used for pore migration while holding the grain growth temperature at $k_{B} T_{g}=0$. At zero grain growth temperature $k_{B} T_{g}=0$, both grain growth and pore migration occur, however, the grain boundary shape is not the minimum energy shape and the grain boundary cannot break 
away from the pores. Miodownik et $\mathrm{al}^{7}$ found similar results for the more general case of pinning phase at a grain boundary rather than at quadra-junctions. They reported at zero grain growth temperature $k_{B} T_{g}=0$, the grain boundary did not have the minimum energy shape.

The simulation temperatures for both, grain growth and pore migration, were varied from $k_{B} T=0.5$ to 3 to study the effect of temperature. As mentioned previously, grain growth was slightly slower at higher Monte Carlo simulation temperatures as illustrated by figure A1. In contrast, when pore migration temperature was held constant at $k_{B} T_{p}=2.0$ and grain growth temperature was varied from $\boldsymbol{k}_{B} \boldsymbol{T}_{g}=0.5$ to 2 , grain growth became faster with increasing grain growth temperature as shown in figure A2a. This contrasting behavior is due to the presence of pores. The data shown in figure A2a is for the case when pores and grains are moving at a constant velocity while maintaining their minimum energy shape. At higher grain growth temperature $k_{B} T_{g}$, there are more fluctuations in the shape of the grain boundary. This noise in the grain boundary relaxes the minimum energy shape of both the grain boundary and the pore. Since the grain boundary and pore do not have to maintain their equilibrium shape so strictly, they can be more responsive to the driving force and thus faster at the high grain growth temperatures. At very high grain growth temperatures $k_{B} T_{g}=2.0$, the grain boundary had sufficient energy to break away from the pores.

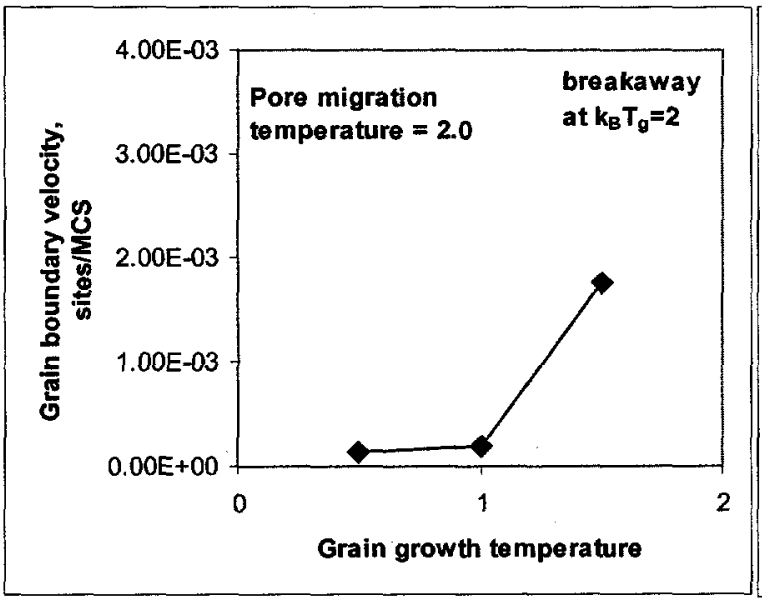

Figure A2a. Grain boundary velocity as a function of grain growth temperature at constant pore migration temperature, $k_{B} T_{p}=2$. The grain boundary broke away from the pores at $k_{B} T_{g}=2$

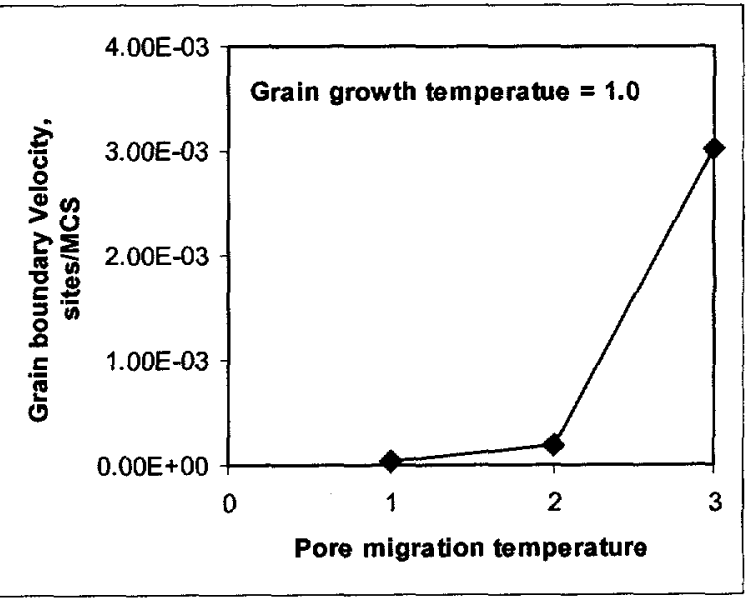

Figure A2b. Grain boundary velocity as a function of pore migration temperature at constant grain growth temperature, $k_{B} T_{g}=1$

When the grain growth temperature was held constant at $\boldsymbol{k}_{B} \boldsymbol{T}_{g}=1$ and pore migration temperature was varied from $\boldsymbol{k}_{B} \boldsymbol{T}_{p}=1.0$ to 3.0 , grain growth became faster with increasing pore migration temperature as shown in figure A2b. The higher grain boundary velocity results from more mobile pores at the higher pore migration temperatures. The increased pore mobility at the higher pore migration temperatures is again attributed to the fact that the pore equilibrium shape is relaxed at the higher 
temperatures. The grain boundary was less likely to break away from the pores at higher pore migration temperatures since pores are mobile at the higher temperatures.

The results presented in this section suggest that finite simulation temperatures must be used for both grain growth and pore migration to correctly simulate grain growth in the presence of a mobile phase. However, any finite temperature below the disordering temperature may be used. There is no threshold temperature above which proper simulation is obtained as observed in some previous Potts model simulations ${ }^{15,19}$.

\section{Acknowledgements}

This work was performed at Sandia National Laboratories, a multiprogram laboratory operated by Sandia Corporation, a Lockheed Martin Company, for the U.S. Department of Energy under contract number DE-AC04-94AL85000.

\section{References:}

\footnotetext{
${ }^{1}$ W.D. Kingery and B. Francois, "Grain Growth in Porous Compacts", J. Am. Ceram. Soc., 48 [10] 546-47 (1965).

${ }^{2}$ R.J. Brook, "Pore-Grain Boundary Interactions and Grain Growth", J. Am. Ceram. Soc., 52[1] 56-57 (1969).

${ }^{3}$ J. Zhoa and M.P. Harmer, "Effect of Pore Distribution on Microstructure Development: II, First- and Second-Generation Pores", J. Am. Ceram. Soc., 71 [7] 530-39 (1988).

4 J. Rödel and A.M. Glaeser, "Pore Drag and Pore-Boundary Separation in Alumina", J. Am. Ceram. Soc., 73 [11] 3302-3312 (1990).

5 V. Tikare and E.A. Holm, "Simulation of Grain Growth and Pore Migration in a Thermal Gradient", J. Am. Ceram. Soc., 81 [3] 480-484 (1998),

${ }^{6}$ M. Hillert, "Inhibition of Grain Growth by Second-Phase Particles", Acta Metall., 36 [12] 3177-3181 (1988).

${ }^{7}$ M. Miodownik, J.W. Martin and A. Cerezo, "Mesoscale simulation of particle pinning”, Phil. Mag. A 79 [1] 203-222 (1999).

${ }^{8}$ F. Y. Wu "The Potts Model," Rev. Modern Phys., 54 [1] 235-268 (1982).

${ }^{9}$ D.J. Srolovitz, G.S. Grest, M.P. Anderson, and A.D. Rollett, "Computer Simulation of Recrystallization II. Heterogeneous Nucleation and Growth," Acta Metall., 36 [8] 2115-2128 (1988).

${ }^{10}$ J. Wejchert, D. Weaire, J.P. Kermode, "Monte Carlo Simulation of the Evolution of a Two-Dimensional Soap Froth,” Phil. Mag. B53 15-24 (1986).

$"$ E.A. Holm, J.A. Glazier, D.J. Srolovitz, G.S. Grest, "Effects of Lattice Anisotropy and Temperature on Domain Growth in the Two-Dimensional Potts Model," Phys. Rev. A, 43 [6] 2662-2668 (1991).

${ }^{12}$ G. Gottstein and L.S. Shvindlerman, "Theory of Grain Boundary Motion in the Presence of Mobile Particles," Acta Metall. Mater. Vol. 41 [11] 3267-3275 (1993).

${ }^{13}$ J.W. Cahn, "The Impurity-Drag Effect in Grain Boundary Motion," Acta Metal. $10789-798$ (1962).

${ }^{14}$ K. Lucke and H.P. Stuwe, "On the Theory of Impurity Controlled Grain Boundary Motion," Acta Metal. 19 1087-1099 (1971)

${ }^{15}$ V. Tikare and J.D. Cawley, "Numerical Simulation of Grain Growth in Liquid Phase Sintered Materials II. Study of Isotropic Grain Growth", Acta Mater. 46 [4] 1343-1356 (1998).

${ }^{16}$ G.N. Hassold, I-W, Chen, D.J. Srolovitz, "Computer Simulation of Final-Stage Sintering : I, Model, Kinetics, and Microstructure," J. Am. Ceram. Soc., 73 [10]2857-64 (1990).

${ }^{17}$ G.S. Grest, S.A. Safran and P.S. Sahni, "Temperture dependence of domain growth", J. Appl. Phys. 55[6] 2432-2434 (1984).

${ }^{18}$ E.A. Holm and M. Miodownik, "Temperature dependence of grain boundary velocity", in press.

${ }^{19}$ T.T. Rautianinen and A.P. Sutton, "Influence of the atomic diffusion mechanism on morphologies, kinetics, and the mechanisms of coarsening during phase separation," Phys. Rev. B. 59 [21] 13681 - 92 (1999).
} 